Journal of Engineering and Applied Sciences 14 (Special Issue 7): 9917-9922, 2019

ISSN: 1816-949X

(C) Medwell Journals, 2019

\title{
Preparation of Manganese Sulfide Thin Film by Thermal Evaporation Technique Deposition: Application of the Experimental Design Photodiode
}

\author{
${ }^{1}$ Mazin H. Hasan, ${ }^{2}$ Fuad T. Ibrahim and ${ }^{3}$ Huda N. Abed \\ ${ }^{1}$ Department of Physics, College of Science, University of Anbar, Baghdad, Iraq \\ ${ }^{2}$ Department of Physics, College of Science, University of Baghdad, Baghdad, Iraq \\ ${ }^{3}$ Al Forat Vocational Preparatory School, Baghdad, Iraq
}

\begin{abstract}
Manganese Sulfide (MnS) thin films were prepared by thermal evaporation technique which deposited on a glass and silicon substrate. The deposited films were examined for their morphology and crystal structure by X-Ray Diffraction (XRD). The MnS heterojunction was successfully fabricated by using thermal evaporation technique at different temperature. The I-V properties of the heterogeneous MnS depend heavily on the structure. Silicon improves performance MnS shows good transparency in the spectral range $300-900 \mathrm{~nm}$ and the electrical properties of the system are highly dependent on the structure. The maximum value of the $\mathrm{R}(\lambda)$ spectral response of the $\mathrm{MnS}$ detection amplifier is $0.165 \mathrm{~A} / \mathrm{W}$ at $450 \pm 50 \mathrm{~nm}$. The maximum detection value of $\mathrm{D}(\lambda)$ was found around $2.359 \times 10^{12}(\mathrm{~cm} \mathrm{~Hz}-1 / \mathrm{W})$ at $450 \pm 60 \mathrm{~nm}$ wavelength of the MnS optical amplifier.
\end{abstract}

Key words: MnS, structural properties, optical properties, AFM, XRD, photodiode

\section{INTRODUCTION}

Manganese sulfide is a broad energy gap $(3.1 \mathrm{eV})$ dilute magnetic semiconductor material that is of potential regard in short wavelength optoelectronic applications such as in photodetector selective coatings, solar cells, sensors, optical mass memories (Eckertova, 1977; Lokhande, 1991). Therefore, the knowledge of the optical properties of thin films is of significance for many applications. Manganese Sulfide (MnS) thin films or powders can be found in several polymorphic forms: the rock-salt type structure (a-MnS) which is the most common form by low temperature growing techniques it crystallizes into the zinc-blende (b-MnS) or wurtzite (c-MnS) structure (Greene et al., 1968; Fan et al., 2003). Interesting electronic properties of manganese attracts a major attention in techno-logical applications. Manganese and its compounds have important semiconducting properties. Recently metal chalcogenide thin film materials have opened a new area in the field of electronic applications. Their properties can be changed by changing the crystallite size and/or thickness of the film depending upon the deposition conditions, the structural, electrical and optical properties of these materials can be controlled in many ways (Greene et al., 1968; O'Brien et al., 2000). Materials containing manganese are interesting because their applications are possible in many areas of modern technology. Manganese Sulfide (MnS) is a magnetic semiconductor material $\left(E_{g}\right.$ $=3.1 \mathrm{eV}$ ) that is of potential interest in short wavelength optoelectronic applications such as in solar selective coatings, solar cells, sensors, photoconductors, optical mass memories (Fan et al., 2003; Tappero et al., 1997; Lokhande et al., 1998). MnS thin films or powders can be found in several polymorphic forms: the rock salt type structure $(\alpha \mathrm{MnS})$ which is the most common form by low temperature growing techniques it crystallizes into the zinc blende $(\beta \mathrm{MnS})$ or wurtzite $(\gamma \mathrm{MnS})$ structure (Clendenen and Drickamer, 2006; Kobayashi et al., 1995). MnS is extensively studied in the literature, preparation of its thin films has been carried out by different methods such as radiofrequency sputtering (Mayen-Hernandez et al., 2003; Oidor-Juarez et al., 2002), hydrothermal (Zhang et al., 2002, 2003; An et al., 2003), molecular beam epitaxy (David et al., 2002) and Chemical Bath Deposition (CBD) (Lokhande et al., 1998; Reddy, 2007). The properties of thin films prepared by different methods are critically dependent on the nature of preparation technique.

In this study, MnS has been fabricated by high vacuum thermal evaporation. Structural, morphology and optical of MnS has been investigated. The XRD measurements disclosed that the MnS was cubic crystal structure. AFM image showed that the produced MnS films have semi spherical shaped with a high homogenous. The energy band gap of MnS films which prepared by thermal evaporation technique has been deter-mined from optical measurements found to be around $3.05 \mathrm{eV}$. Dark and illuminated current-Voltage I-V characteristics, spectral responsivity, specific

Corresponding Author: Fuad T. Ibrahim, Department of Physics, College of Science, University of Baghdad, Baghdad, Iraq 
detectivity and minority carrier lifetime of photodiode relive that the $\mathrm{MnS}$ can be used as a photodiode application.

\section{MATERIALS AND METHODS}

Experimental work: The glass and silicon chip were used as substrate materials for deposition of thin $\mathrm{MnS}$ films. The glass slides were cut into $1.5 \times 1.5 \mathrm{~cm}$ compatible with the dimension of the substrate holder using a steel cutting tool. The glass slides were thoroughly cleaned prior to the deposition process to reach the reasonable adhesion coefficient as followed in the following procedure. The glass slides were first cleaned using a diluted solution of chemical detergents to remove impurities and protein substances on the surface of the slides. Si samples were then cleaned with alcohol and an ultrasonic bath to remove impurities and debris from its surface. These substrates were drilled with HF (10\%) for 5 min to remove the original oxide. The bottom electrode is coated with a thick aluminum layer prior to the anode process and electrical properties are measured. They are obtained under vacuum aluminum wire with high purity (99.99\%). Evaporation process started at 10-5 torr pressure. The best state of good communication was met with a layer of $200 \mathrm{~nm}$. The Manages Sulfide (MnS) deposited by thermal evaporation technique using coating unit in vacuum about $2 \times 10^{-5}$ torr and put it in a special evaporation molybdenum boat. The rate of evaporation was $23.5 \mathrm{~nm} / \mathrm{min}$ and the film = thickness (400 nm) was measured by interference method. The substrate glass was placed directly above the source at a distance about $18 \mathrm{~cm}$ after cleaned the glass and the this film which deposited one study the structural, topography and optical properties of all films (as-prepared and $150^{\circ} \mathrm{C}$ ) were investigated separately by means of (CuK $\left.\alpha\right)$ XRD-6000, Shimadzu X-ray diffractometer, Fourier transformation infrared spectroscopy, JEOL (JSM-5600) scanning electron microscopy, Philips CM10 pw 6020 transmission electron microscopy, Angstrom AA3000 Atomic Force Microscopy and Cary 100 Conc plus $\mathrm{UV}-\mathrm{V}$ is spectrophotometer. Infrared spectroscopy (FTIR-8400S) and Cary 100 Conc plus UV-Vis spectrophotometer.

\section{RESULTS AND DISCUSSION}

Figure 1 show the XRD different pattern of prepped thin films by thermal evaporation technique and deposited on glass substrate than annealing at tow temperature (as-prepared and $150^{\circ} \mathrm{C}$ ). Figure 1 revels a strong peak of $\mathrm{MnS}$ (as prepared and $150^{\circ} \mathrm{C}$ ) at $2 \theta\left(29.66^{\circ}, 29.692^{\circ}\right.$ ), respectively only along the (111) direction at annealing temperature, correspond ASTM. All the diffraction peaks in Fig. 1 are indicates to cubic structure with no trail of hexagonal or other faces. The crystalline size $\mathrm{C}$ for a knowing by $\mathrm{X}$-ray wavelength $\lambda$ at the diffraction angle $\theta$ from Eq. 1 as given by Eckertova (1977):

$$
\mathrm{C}=\alpha \lambda / \mathrm{FWHM} \cos (\theta)
$$

Where:

FWHM : The Full Width at the Half Maximum of the characteristic spectrum in units of radians and $\lambda(\mathrm{nm})$

$\alpha \quad$ : The Scherrer constant ( $1>a>0.89)$, fund to be (10.3 nm) which is agreement with the determined AFM in visitation

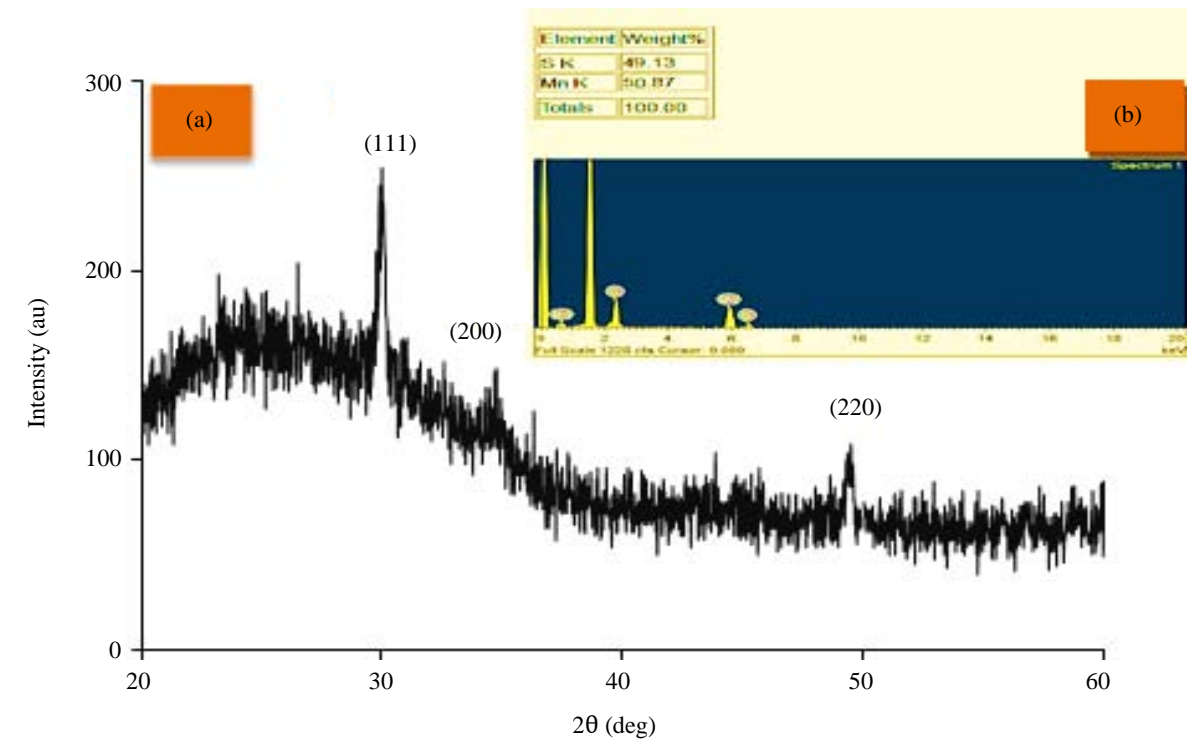

Fig. 1: (a) XRD spectra of MnS films and (b) MnS EDX 

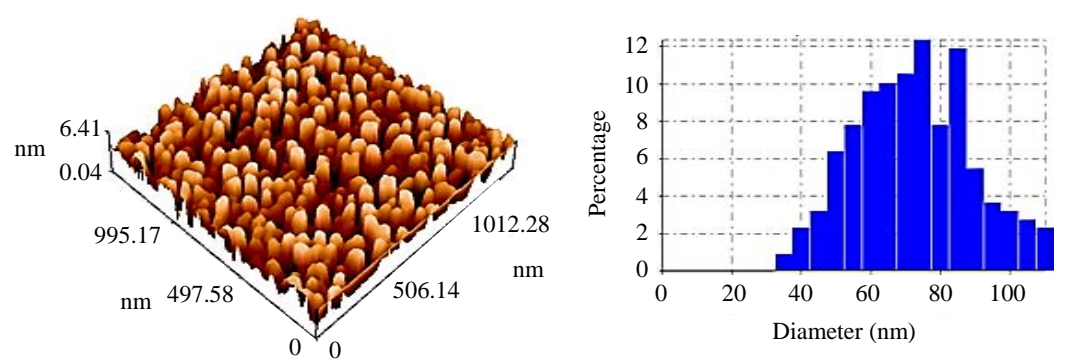

Fig. 2: 3D AFM images of MnS and granularity accumulation distribution chart

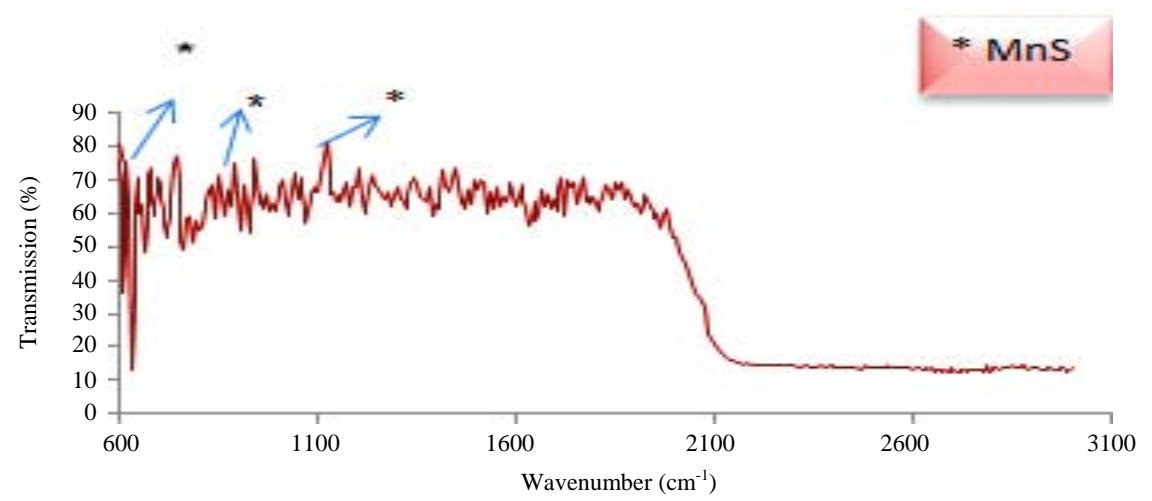

Fig. 3: FTIR spectra of MnS thin films

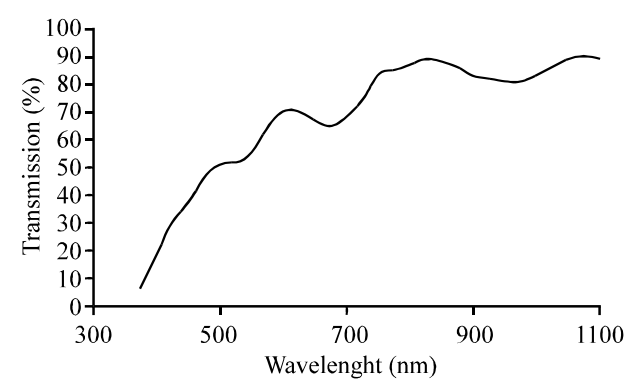

Fig. 4: Optical transmittance of MnS thin films

Table 1: Average grain, roughness density and RMS of thin films

\begin{tabular}{lc}
\hline Samples & MnS (nm) \\
\hline Average GS & 70.01 \\
Roughness & 2.01 \\
RMS & 2.37 \\
\hline
\end{tabular}

Figure 2 shows 3D AFM image and granularity accumulation distribution chart of MnS nanostructure prepared by thermal evaporation technique on glass substrate. Substrate is well covered with MnS nanostructure; distributed uniformly on the surface. It is obvious from Fig. 2 that the nanostructure has small ordered particles with semispherical shape. The average particle size estimated with the aid of software (Table 1).

Figure 3 shows the FTIR spectra of MnS: Al Sample which showed several significant absorption peaks. The broad absorption band in the region of $600-2100 \mathrm{~cm}^{-1}$ is assigned to MnS stretching mode .The spectra of $\mathrm{MnS}$ showed the absorption bands at 620,892 and $1123 \mathrm{~cm}^{-1}$ in control and treated sample, attributing to -OH stretching vibration.

Figure 4 shows displays the transmission as a function of wavelength of MnS thin films is which prepared by thermal evaporation techniques and deposited on glass substrate. It is obvious that the film gives good transparency characteristics at the spectral range $600-1000 \mathrm{~nm}$. The date is corrected for glass in UV-regain, the transmission is sharply increases because of the wide of absorbed particle size. Also, the maximum value of transmittance $(90.1 \%)$ that is indicated as window on solar cell application at rang to N-IR or the material (MnS) can be describe in absorber material below $550 \mathrm{~nm}$. The optical energy gap of MnS was calculated by the relation (Lokhande et al., 1998):

$$
\alpha h v=A(h v-E g)^{n}
$$

Where:

Aa $=$ The constant

$v=$ The transition frequency and the exponent $n$ characterizes the nature of band transition

The $n=1 / 2$ and $n=3 / 2$ corresponds to direct allowed and direct forbidden transitions and $\mathrm{n}=2$ and 3 corresponds to indirect allowed and indirect forbidden 


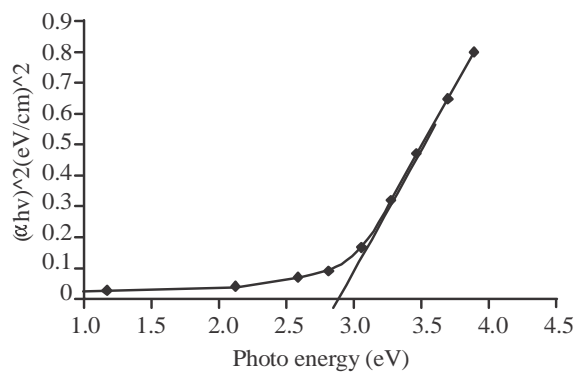

Fig. 5: Plot of $(\alpha h v)^{2}$ versus hv curve of as-prepared MnS thin films

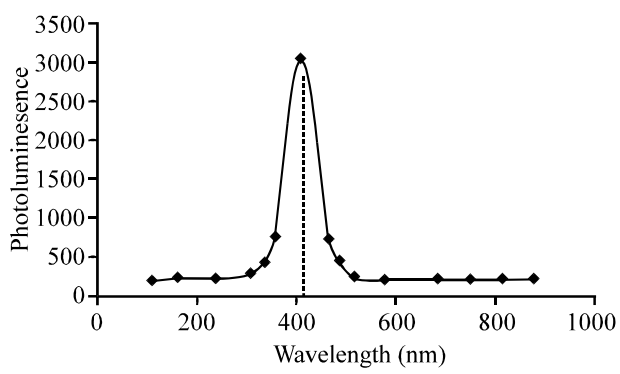

Fig. 6: PL spectra of MnS

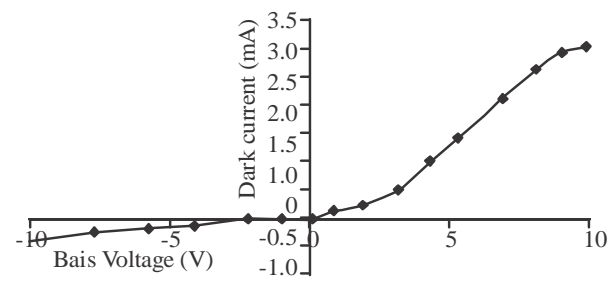

Fig. 7: Characteristic under forward reverse bias of the MnS for MnS

transitions, respectively. Figure 5 shows the band gap of MnS thin films is which prepared by thermal evaporation techniques and deposited on glass substrate. Measured from the plot of the square of $(\alpha h v)^{2}$ versus photon energy $\mathrm{h} v$ (where $\alpha$ is the absorption coefficient) by extrapolating the linear part of the curve toward the photon energy axis. The optical energy gap of MnS thin film is found to be around $2.85 \mathrm{eV}$, this values are agree with the values obtained by Reddy et al. (2007).

Figure 6 shows the PL emission spectra of MnS prepared by thermal evaporation technique room temperature with an excitation source of wavelength of $400 \mathrm{~nm}$, single sharp broad emission peak centered at the $3000 \mathrm{~nm}$.

Figure 7 shows dark characteristics of $\mathrm{I}-\mathrm{V}$ in the anterior and reverse direction of heterozygous cells $\mathrm{MnS}$. The forward current of the heterojunction is very small at a voltage lower than $1.2 \mathrm{~V}$. The current is known as the

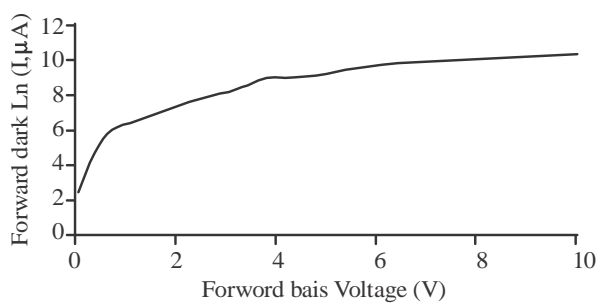

Fig. 8: Variation of $\ln (\mathrm{I})$ with bias voltage of $\mathrm{MnS}$ heterojunction

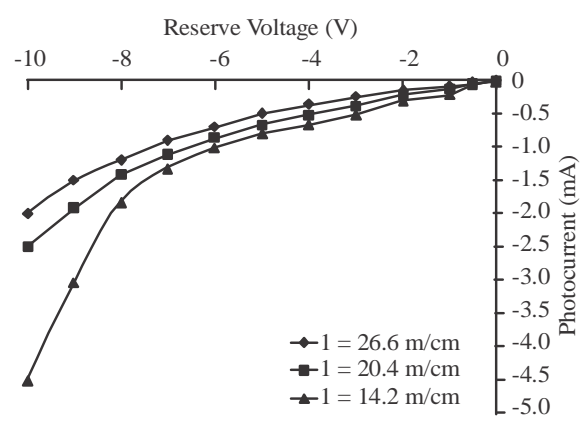

Fig. 9: Characteristic under forward reverse bias for $\mathrm{MnS}$

recirculation current at low voltages only. It is created when each excited electron forms the equivalence range of the conductive band. The second high voltage zone represents the area of propagation or bending which depends on serological resistance. In this region; the bias voltage can deliver electrons with enough energy to penetrate the barrier between the two sides of the junction.

Figure 8 shows that the variation of $\ln (\mathrm{I})$ with bias voltage of MnS heterojunction. The ideality factors of heterojunctions are estimated Ideality factor was found to be around 4.8.

Figure 9 shows that the characteristics of the inverse current of the measured instrument in the light current under the tungsten lamp illumination 26.6, 20.4 and $14.2 \mathrm{~W} / \mathrm{cm}^{2}$. It can be observed that the inverse current value in a standard voltage of hetero-diode meters under luminance is higher than that in the dark and can be seen from Fig. 9 that the current value in a certain voltage of the opposite connectors under the illumination is higher than in the darkness, generation of the current caused by the carrier due to electron-hole production due to light absorption. This behavior produces useful information about electron hole pairs which are effectively generated in the link by the photons that occur.

Figure 10 shows a linear relation between inverse of square of Capacitance $\left(1 / \mathrm{C}^{2}\right)$ reverse bias voltage for $\mathrm{MnS}$, indicating abrupt junction. The built-in potential $\mathrm{V}_{\mathrm{bi}}$ 


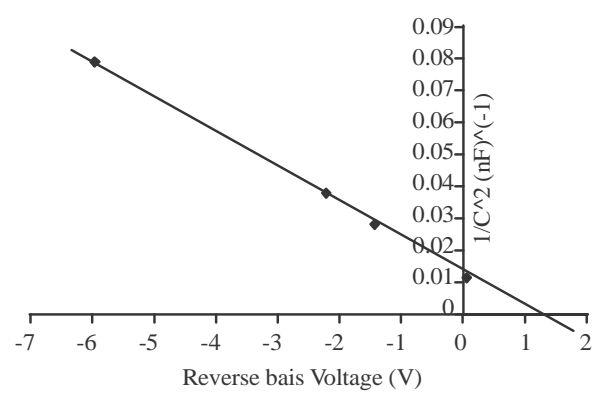

Fig. 10: The $1 / \mathrm{C}^{2}$ versus reverse voltage of $\mathrm{MnS}$ photodieode

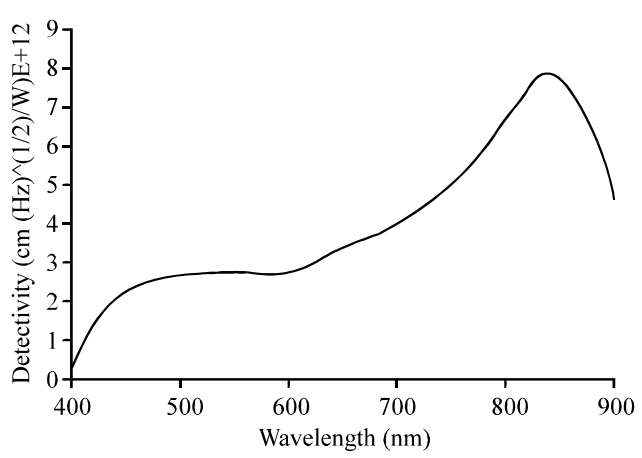

Fig. 11: Spectral detectivity as a function of wavelength of $\mathrm{MnS}$ photodiode

value were obtained after extrapolating the $\left(1 / C^{2}\right)$ point to the voltage axis. The built-in potential $\mathrm{V}_{\mathrm{bi}}$ for $\mathrm{MnS}$ was $1.2 \mathrm{~V}$.

Figure 11 shows that the spectrum detectivity curve in $\mathrm{MnS}$ consists of two reaction peaks; the first peak is at $450 \mathrm{~nm}$ due to the absorption margin of $\mathrm{MnS}$ nanoparticles while the second is located at $800 \pm 50 \mathrm{~nm}$ as a result of absorption of the silicon edge.

\section{CONCLUSION}

The MnS heterojunction was successfully fabricated by using thermal evaporation technique at different temperature. The I-V characteristics of heterojunction $\mathrm{MnS}$ are strongly dependent on the structure. The silicon improves the performance MnS show a good transparency in the spectral range 400-900 nm and the electrical characteristics of heterojunction are strongly dependent on the structure. The maximum value of spectral Responsivity $\mathrm{R}(\lambda)$ of $\mathrm{MnS}$ photodetector is around $0.165 \mathrm{~A} / \mathrm{W}$ at $450 \pm 50 \mathrm{~nm}$. The maximum value of the specific Detectivity $D(\lambda)$ is found to be $2.359 \times 10^{12} \mathrm{~W}^{-1} \mathrm{~cm} \mathrm{~Hz}^{-1}$ located at $450 \pm 50 \mathrm{~nm}$ wavelength for $\mathrm{MnS}$ photodetector.

\section{REFERENCES}

An, C., K. Tang, X. Liu, F. Li and G. Zhou et al., 2003. Hydrothermal preparation of $\alpha$-MnS nanorods from elements. J. Cryst. Growth, 252: 575-580.

Clendenen, R.L. and H.G. Drickamer, 2006. Lattice parameters of nine oxides and sulfides as a function of pressure. J. Chem. Phys., 44: 4223-4228.

David, L., C. Bradford, X. Tang, T.C.M. Graham and K.A. Prior et al., 2002. Growth of zinc blende MnS and $\mathrm{MnS}$ heterostructures by MBE using $\mathrm{ZnS}$ as a sulphur source. Proceedings of the International Conference on Molecular Bean Epitaxy, September 15-20, 2002, IEEE, San Francisco, California, USA., pp: 261-262.

Eckertova, L., 1977. Physics of Thin Films. Plenum Press, New York, USA., ISBN-13: 978-1-4615-7591-7, Pages: 254.

Fan, D., X. Yang, H. Wang, Y. Zhang and H. Yan, 2003. Photoluminescence of MnS thin film prepared by chemical bath deposition. Phys. B. Condens. Matter, 337: 165-169.

Greene, R.L., D.D. Sell, R.S. Feigelson, G.F. Imbusch and H.J. Guggenheim, 1968. Impurity-induced optical fluorescence in MnF2. Phys. Rev., 171: 600-610.

Kobayashi, M., T. Nakai, S. Mochizuki and N. Takayama, 1995. Validity of the Sugano-Tanabe diagram for band states in $\mathrm{MnO}$ and $\mathrm{MnS}$ under high pressure. J. Phys. Chem. Solids, 56: 341-344.

Lokhande, C.D., 1991. Chemical deposition of metal chalcogenide thin films. Mater. Chem. Phys., 27: 1-43.

Lokhande, C.D., A. Ennaoui, P.S. Patil, M. Giersig and M. Muller et al., 1998. Process and characterisation of chemical bath deposited manganese sulphide (MnS) thin films. Thin Solid Films, 330: 70-75.

Mayen-Hernandez, S.A., S. Jimenez-Sandoval, R. Castanedo-Perez, G. Torres-Delgado and B.S. Chao et al., 2003. Preparation and characterization of polycrystalline MnS thin films by the RF-sputtering technique above room temperature. J. Cryst. Growth, 256: 12-19.

O’Brien, P., D.J. Otway and D. Smyth-Boyle, 2000. The importance of ternary complexes in defining basic conditions for the deposition of $\mathrm{ZnS}$ by aqueous chemical bath deposition. Thin Solid Films, 361: 17-21.

Oidor-Juarez, I., P. Garciia-Jimenez, G. Torres-Delgado, R. Castanedo-Perez and O. Jimenez-Sandoval et al., 2002. Substrate temperature effects on the growth and properties of $\gamma$-MnS thin films grown by RF sputtering. Mater. Res. Bull., 37: 1749-1754. 
Reddy, D.S., D.R. Reddy, B.K. Reddy, A.M. Reddy and K.R. Gunasekhar et al., 2007. Annealing effect on physical properties of thermally evaporated MnS nanocrystalline films. J. Optoelectron. Adv. Mater., 9: 2019-2022.

Tappero, R., P. D'Arco and A. Lichanot, 1997. Electronic structure of a-MnS (alabandite): An AB initio study. Chem. Phys. Lett., 273: 83-90.
Zhang, Y., H. Wang, B. Wang, H. Xu and H. Yan, 2003. Hydrothermal synthesis of metastable $\gamma$-manganese sulfide crystallites. Opt. Mater., 23: 433-437.

Zhang, Y., H. Wang, B. Wang, H. Yan and M. Yoshimura, 2002. Low-temperature hydrothermal synthesis of pure metastable $\gamma$-manganese sulfide (MnS) crystallites. J. Cryst. Growth, 243: 214-217. 